\title{
Epitaxially grown MnAs/GaAs lateral spin valves
}

\author{
D. Saha, M. Holub, P. Bhattacharya, ${ }^{a)}$ and Y. C. Liao \\ Solid-State Electronics Laboratory, Department of Electrical Engineering and Computer Science, University \\ of Michigan, 1301 Beal Avenue, Ann Arbor, Michigan 48109-2122
}

(Received 23 June 2006; accepted 22 August 2006; published online 3 October 2006)

\begin{abstract}
The authors report magnetoresistance of lateral spin valves fabricated from an epitaxially grown MnAs/GaAs heterostructure and utilizing a Schottky tunnel barrier for efficient spin injection. A coercive field difference between the two ferromagnetic MnAs contacts is obtained by a difference in aspect ratio. Peak magnetoresistances of $3.6 \%$ at $10 \mathrm{~K}$ and $1.1 \%$ at $125 \mathrm{~K}$ are measured for a $0.5 \mu \mathrm{m}$ channel length spin valve. The authors observe an exponential decay of the peak magnetoresistance with increasing channel length, which is indicative of diffusive spin transport. The magnetoresistance increases with increasing bias and with decreasing temperature. Control experiments have been carried out to confirm the spin-valve effect. () 2006 American Institute of Physics. [DOI: 10.1063/1.2358944]
\end{abstract}

Efficient electrical injection of spin-polarized carriers into semiconductors from a ferromagnetic contact is a very challenging research field. ${ }^{1,2}$ Spin injection in a lateral geometry is favored over more ubiquitous vertical structures used in tunneling magnetoresistance devices ${ }^{3,4}$ since lateral structures offer a larger degree of freedom in their fabrication and the possibility of integration with other conventional lateral semiconductor devices. All-metal lateral spin valves fabricated by electron-beam lithography have already been demonstrated; ${ }^{5,6}$ however, semiconductor-based lateral spin valves with ferromagnetic (FM) contacts serving as the injector and collector have remained elusive. ${ }^{7-9}$ In this letter we provide evidence of spin injection and collection in a hybrid ferromagnet-semiconductor heterostructure with a lateral device geometry. The variation of magnetoresistance (MR) with channel length, bias, and temperature has been studied in detail. The results are explained in the framework of spin injection, transport, and collection along with micromagnetic simulations.

The spin-valve heterostructures were grown by molecular-beam epitaxy on semi-insulating GaAs(001) substrates [Fig. 1(a)]. A $150 \mathrm{~nm}$ Si-doped $n$-GaAs channel layer was grown at $600{ }^{\circ} \mathrm{C}$, followed by a $30 \mathrm{~nm}$ graded doping $n^{+}$-GaAs contact layer grown at the same temperature, after which the substrate temperature was ramped down for FM MnAs deposition. The doping concentration $\left(N_{D}\right)$ profile along the vertical cross section as estimated from growth conditions is shown in Fig. 1(a). The narrow heavily doped region beneath MnAs creates a triangular tunnel contact, increasing the spin-injection efficiency. ${ }^{10,11}$ The channel doping $\left(N_{D}=1 \times 10^{17} \mathrm{~cm}^{-3}\right)$ was optimized to ensure a large spin-relaxation time at low temperatures. ${ }^{12}$ It is well known that two orientations of MnAs may be realized on $\mathrm{GaAs}(001)$ substrates depending on the growth conditions during nucleation. ${ }^{13}$ For the present work, we selected an As-rich template for growth of a $90 \mathrm{~nm}$ type-A MnAs film, which yields the following epitaxial relationship: $[\overline{1} 120] \mathrm{MnAs} \|[110] \mathrm{GaAs}$ and $[0001] \mathrm{MnAs} \|[\overline{1} 10] \mathrm{GaAs}$. A very low growth rate $(10 \mathrm{~nm} / \mathrm{h})$ and substrate temperature $\left(200{ }^{\circ} \mathrm{C}\right)$ were used during growth of the first few monolay-

${ }^{a)}$ FAX: (734) 763-9324; electronic mail: pkb@eecs.umich.edu ers of MnAs. After the nucleation phase, the growth rate and substrate temperature were both increased to $40 \mathrm{~nm} / \mathrm{h}$ and $250{ }^{\circ} \mathrm{C}$, respectively. An in situ anneal under an $\mathrm{As}_{4}$ flux was performed at $350{ }^{\circ} \mathrm{C}$ to improve the structural and magnetic properties of the MnAs film. The thickness of the MnAs film. $(90 \mathrm{~nm})$ was chosen appropriately so as to maximize the saturation magnetization and suppress the formation of cracks which complicate device fabrication. Superconducting quantum interference device (SQUID) magnetometry measurements yield coercivities of 210 Oe along the easy magnetization axis [110] and $\sim 2.8 \mathrm{~T}$ along the hard axis [110] of an as-grown unpatterned sample, which are comparable to those reported by others. ${ }^{14,15}$

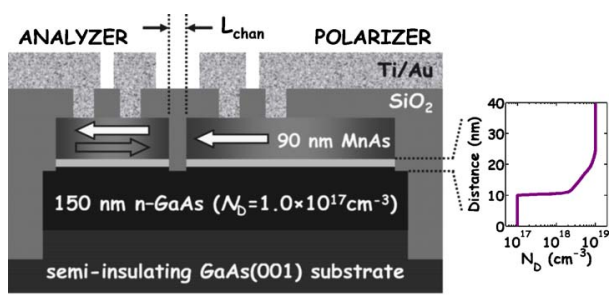

(a)

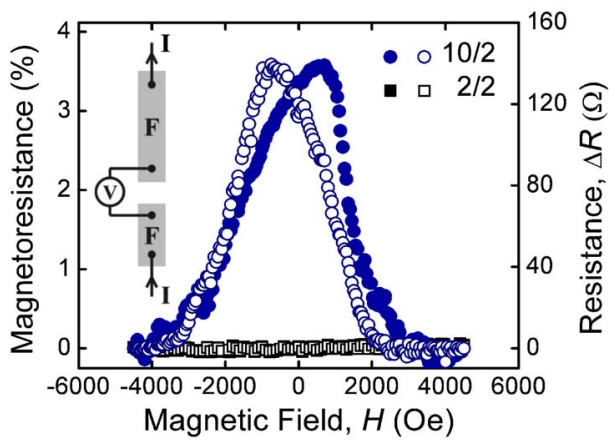

(b)

FIG. 1. (Color online) (a) Schematic cross section of a MnAs/GaAs lateral spin valve. (b) Magnetoresistance and resistance changes at $10 \mathrm{~K}$ as a function of applied magnetic field for a $0.5 \mu \mathrm{m}$ channel length conventional spin valve biased at $5.5 \mathrm{~mA}$. The spin valve is shown schematically in the inset. The legend indicates the length-to-width aspect ratio for the polarizer/ analyzer. Open and closed symbols represent the cases of descending and ascending magnetic fields, respectively. 
Lateral spin valves of channel lengths $L_{\text {chan }}=0.5,0.7$, 1.0 , and $1.5 \mu \mathrm{m}$ with polarizer $(P)$ and analyzer $(A)$ contact length-to-width aspect ratios $(L / W)$ of 10 and 2, respectively, were fabricated using conventional wet and dry etching, photolithography, and metallization techniques. SQUID magnetometry measurements as well as finite difference micromagnetic simulations ${ }^{16}$ using parameters optimized for our growth conditions confirm that a coercive field difference is readily obtained by varying the $\mathrm{MnAs}$ contact aspect ratio. The following three control devices were also fabricated to conclusively confirm spin-valve behavior in devices with different contact aspect ratios: (1) symmetric devices with $L_{\text {chan }}=0.5 \mu \mathrm{m}$ and identical aspect ratios for both contacts $\left[(L / W)_{A}=(L / W)_{P}=2\right],(2)$ ferromagnetic MnAs/nonmagnetic GaAs/nonmagnetic GaAs $(F / N / N)$ lateral devices with $L_{\text {chan }}=0.5 \mu \mathrm{m}$, and (3) nonlocal ${ }^{5}$ spin valves with $L_{\text {chan }}$ $=1 \mu \mathrm{m}$. Devices identical to the lateral spin valves without the nonmagnetic GaAs channel are also fabricated to estimate the contribution from anisotropic magnetoresistance (AMR) to the overall MR. The spin-valve devices operate on the principle that the polarizer, maintained at a negative bias with respect to the analyzer, injects electron spins predominantly of one spin orientation, which are subsequently detected by the analyzer. The polarizer and analyzer efficiencies change to a different extent with changing magnetic field $(H)$ by virtue of their different coercivities, which is manifested as a change in resistance.

The magnetoresistance, defined as $\operatorname{MR}(H)=\left[R_{\uparrow \uparrow}\left(H_{\text {sat }}\right)\right.$ $\left.-R_{\uparrow \downarrow}(H)\right] / R_{\uparrow \uparrow}\left(H_{\text {sat }}\right)$, of the spin valves and control devices were measured for various $L_{\text {chan }}$, temperatures $(T)$, and bias conditions $\left(I_{B}\right)$ using a standard four-probe ac lock-in technique. Figure 1(b) shows MR for spin valves having different (asymmetric) and identical (symmetric) aspect ratios of the FM contact pads with $L_{\text {chan }}=0.5 \mu \mathrm{m}$ at $T=10 \mathrm{~K}$ and $\mathrm{I}_{B}$ $=5.5 \mathrm{~mA}$. The MR for the asymmetric device peaks at $3.6 \%$ for $|H| \sim 700$ Oe in both directions of the magnetic field sweep. The MR peaks correspond to antiparallel magnetization of the MnAs pads arising from their coercive field difference. The change of MR with magnetic field is gradual, and the change is very large even before there is any substantial change in net contact magnetization. Micromagnetic simulations of our device and the orientation of magnetic domains at the edge of the FM contacts, which are the domains responsible for spin injection, at different points of the corresponding hysteresis are shown in Fig. 2. It is found that the domains at the very near edge of the channel start responding to $H$ much earlier (even before $H=0$ ) ${ }^{17}$ but owing to their small contribution to the overall magnetization it is not observable in the hysteresis data. This explains the discrepancy between the MR plot and SQUID magnetization data. Furthermore, the gradual rotation of domains explains the gradual change in MR with $H$. There is no noticeable magnetoresistance (peak $\mathrm{MR} \sim 0.04 \%$ and $\Delta R_{\text {peak }} \sim 3.5 \mathrm{~m} \Omega$ at $H=0$ ) for the symmetric device which demonstrates the spin-valve effect in our devices. The MR for the $F / N / N$ lateral devices is also found to be negligible (not shown) which further confirms that the MR in our devices is due to the spin-valve effect only. The measured MR of the nonlocal spin valve as a function of magnetic field is shown in Fig. 3(a). The nonlocal spin valve removes the AMR contribution and the Hall effects of the ferromagnetic electrodes. ${ }^{5}$ The MR for the nonlocal spin valves show a similar change with

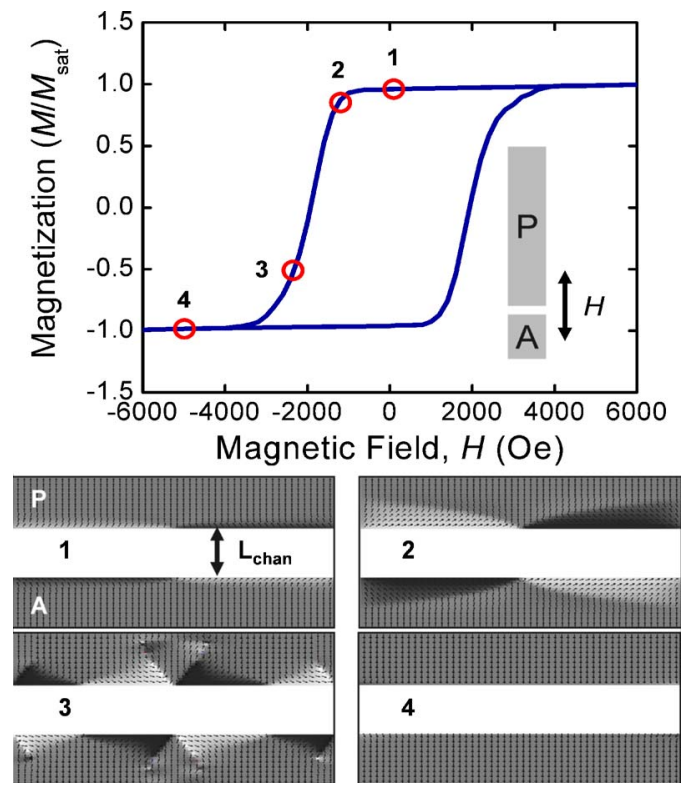

FIG. 2. (Color online) Simulated magnetic domain patterns for a MnAs/GaAs spin valve at different positions along the hysteresis loop. A zoomed view of the domain patterns near the channel is shown for each case. The inset shows a schematic depiction of the simulated spin valve with a $10 \times 5 \mu \mathrm{m}^{2}$ analyzer $(A)$ and $50 \times 5 \mu \mathrm{m}^{2}$ polarizer $(P)$.

varying magnetic field as the conventional spin valve. A $\Delta R_{\text {peak }} \approx 66 \Omega$ was measured for a $L_{\text {chan }}=1 \mu \mathrm{m}$ nonlocal spin valve under the same operating conditions as for the conventional spin valve. This value is 2.3 times smaller, compared to the theoretically predicted factor of 2 for a transparent barrier, ${ }^{5}$ than the value measured for a similar conventional spin valve. We have not observed any difference between the shapes of the $\Delta R$ curves in the two cases, which indicates that any stray AMR contribution that might be present is too small to appear considering the magnitude of the MR measured in the above cases. To precisely estimate the anomalous AMR contribution, MR measurements were done on a strip of MnAs contact $(L / W=12)$ fabricated identically to the conventional spin valve but without a GaAs channel. Figure 3(b) shows the MR for such a device under identical bias conditions as for the conventional spin valve. The measured $\Delta R_{\text {peak }} \approx 3 \mathrm{~m} \Omega$ at zero magnetic field is five orders of magnitude smaller than the value measured for the spin valve. This explains the similarity of the shape of the curves observed in conventional and nonlocal spin valves. The near-zero response [Fig. 1(b)] measured for the symmet-
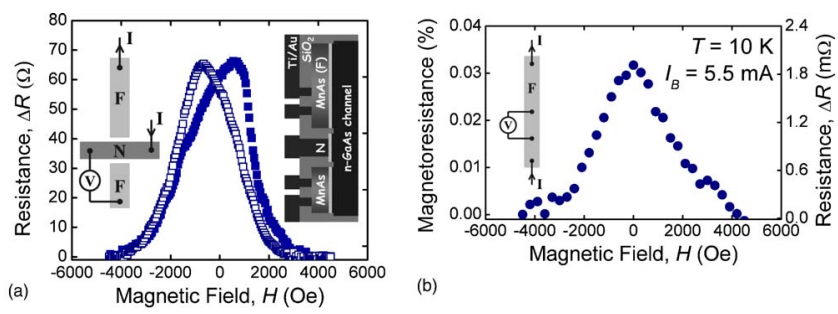

FIG. 3. (Color online) (a) Resistance change as a function of applied magnetic field for a $1 \mu \mathrm{m}$ channel length nonlocal spin valve. Top- and sideview schematics of the nonlocal spin valve are shown in the inset. Open and closed symbols represent the cases of descending and ascending magnetic fields, respectively. (b) Magnetoresistance and resistance changes due to stray effects of a single MnAs strip contact, which is shown schematically in the inset. 
(a)

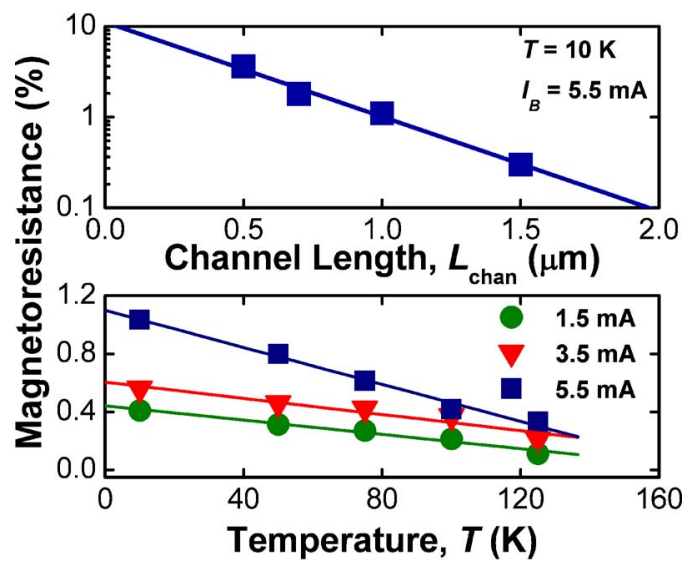

(b)

FIG. 4. (Color online) (a) Peak magnetoresistance vs channel length measured at $10 \mathrm{~K}$. (b) Peak magnetoresistance vs temperature for a $1 \mu \mathrm{m}$ spin valve with applied biases of $I_{B}=1.5,3.5$, and $5.5 \mathrm{~mA}$. The lines represent a linear least squares fit to the data.

ric spin valves indicates that the MR in these devices result from stray effects.

Figure 4(a) shows that the peak MR for the conventional spin valve varies exponentially with $L_{\text {chan }}$, which indicates that spin transport is diffusive in the channel. ${ }^{18,19}$ As $L_{\text {chan }}$ is decreased, the number of spin-polarized electrons that reach the analyzer before spin relaxation increases, yielding a larger peak MR. Figure 4(b) shows the temperature dependence of the peak MR under different bias voltages. Five spin valves across various grown and processed samples with $L_{\text {chan }}=1.0 \mu \mathrm{m}$ were measured under identical experimental conditions, and the results are highly reproducible. The peak MR increases with decreasing temperature for a fixed bias voltage due to increase in average spin-relaxation time and hence spin-flip length. The increase in MR with bias, contrary to theoretical predictions, ${ }^{20,21}$ can be explained by considering the effect of spin injection through a tunnel barrier at higher bias values. The depletion region width for the electrons to tunnel through decreases with increasing bias, leading to efficient spin injection from the polarizer. In contrast the analyzer efficiency does not change much with bias. This leads to the overall increase in MR at high bias. However, MR values higher than those shown in Fig. 4(b) could not be attained since most of our devices suffered breakdown for an applied current bias greater than $5.5 \mathrm{~mA}$.

Expected spin injection and MR values at $T=10 \mathrm{~K}$ and $I_{B}=5.5 \mathrm{~mA}$ for a $0.5 \mu \mathrm{m}$ channel length spin valve, calculated using the simple diffusive injection model in Ref. 18 with experimentally determined values for the model parameters and appropriate value for bulk spin polarization $(\beta)$ from Ref. 15 , are found to be $0.04 \%$ and $<0.01 \%$. The value of MR $(\sim 3.6 \%)$ measured in this study is much larger, which indicates efficient spin-polarized carrier injection by tunneling through the Schottky barrier in our devices. Large spin-injection efficiencies of $32 \%$ have also been reported for similar Fe/AlGaAs Schottky tunnel barrier contacts as determined from the electroluminescence polarization of spinpolarized light-emitting diodes. ${ }^{22}$

In summary, we have conclusively demonstrated the spin-valve effect in a lateral MnAs/GaAs device and have investigated the temperature, bias, and channel length dependence of the observed magnetoresistance. A peak magnetoresistance of $3.6 \%$ was observed at $10 \mathrm{~K}$ in a $0.5 \mu \mathrm{m}$ channel length spin valve, and a finite magnetoresistance can be seen for temperatures up to $125 \mathrm{~K}$. These results provide experimental verification of spin injection and collection in a lateral structure and can serve as a benchmark for future spinbased devices.

This work is supported by the Office of Naval Research under Grant No. N00014-06-1-0025. The authors gratefully acknowledge $\mathrm{Z}$. $\mathrm{Wu}$ for assistance with device fabrication.

${ }^{1}$ B. T. Jonker, Proc. IEEE 91, 727 (2003).

${ }^{2}$ Semiconductor Spintronics and Quantum Computation, edited by D. D. Awschalom, N. Samarth, and D. Loss (Springer, Berlin, 2002).

${ }^{3}$ J. S. Moodera, L. R. Kinder, T. M. Wong, and R. Meservey, Phys. Rev. Lett. 74, 3273 (1995).

${ }^{4}$ T. Miyazaki and N. Tezuka, J. Magn. Magn. Mater. 139, L231 (1995).

${ }^{5}$ F. J. Jedema, M. S. Nijboer, A. T. Filip, and B. J. van Wees, Phys. Rev. B 67, 085319 (2003).

${ }^{6}$ T. Kimura, J. Hamrle, Y. Otani, K. Tsukagoshi, and Y. Aoyagi, J. Magn. Magn. Mater. 286, 88 (2005).

${ }^{7}$ P. R. Hammar, B. R. Bennett, M. J. Yang, and M. Johnson, Phys. Rev. Lett. 83, 203 (1999).

${ }^{8}$ W. Y. Lee, S. Gardelis, B.-C. Choi, Y. B. Xu, C. G. Smith, C. H. W. Barnes, D. A. Ritchie, E. H. Linfield, and J. A. C. Bland, J. Appl. Phys. 85, 6682 (1999).

${ }^{9}$ S. Hacia, T. Last, S. F. Fischer, and U. Kunze, J. Supercond. 16, 187 (2003).

${ }^{10}$ E. I. Rashba, Phys. Rev. B 62, R16267 (2000).

${ }^{11}$ J. D. Albrecht and D. L. Smith, Phys. Rev. B 68, 035340 (2003).

${ }^{12}$ R. I. Dzhioev, K. V. Kavokin, V. L. Korenev, M. V. Lazarev, B. Ya. Meltser, M. N. Stepanova, B. P. Zakharchenya, D. Gammon, and D. S. Katzer, Phys. Rev. B 66, 245204 (2002).

${ }^{13}$ M. Tanaka, Semicond. Sci. Technol. 17, 327 (2002), and references therein.

${ }^{14}$ M. Tanaka, J. P. Harbison, M. C. Park, Y. S. Park, T. Shin, and G. M. Rothberg, Appl. Phys. Lett. 65, 1964 (1994).

${ }^{15}$ R. P. Panguluri, G. Tsoi, B. Nadgorny, S. H. Chun, N. Samarth, and I. I. Mazin, Phys. Rev. B 68, 201307(R) (2003).

${ }^{16} \mathrm{M}$. Donahue and D. Porter, http://math.nist.gov/oommf

${ }^{17}$ S. T. Chui, IEEE Trans. Magn. 34, 1000 (1998).

${ }^{18}$ G. Schmidt, D. Ferrand, L. W. Molenkamp, A. T. Filip, and B. J. van Wees, Phys. Rev. B 62, R4790 (2000).

${ }^{19}$ Z. G. Yu and M. E. Flatté, Phys. Rev. B 66, 235302 (2002).

${ }^{20}$ Y. Y. Wang and M. W. Wu, Phys. Rev. B 72, 153301 (2005).

${ }^{21}$ M. E. Flatté, J. M. Byers, and W. H. Lau, Semiconductor Spintronics and Quantum Computation, edited by D. D. Awschalom, N. Samarth, and D. Loss (Springer, Berlin, 2002), Chap. 4, pp. 140 and 141.

${ }^{22}$ A. T. Hanbicki, B. T. Jonker, G. Itskos, G. Kioseoglou, and A. Petrou, Appl. Phys. Lett. 80, 1240 (2002). 